\title{
BETWIXT TURING AND KLEENE
}

\author{
DAG NORMANN AND SAM SANDERS
}

\begin{abstract}
Turing's famous 'machine' model constitutes the first intuitively convincing framework for computing with real numbers. Kleene's computation schemes S1-S9 extend Turing's approach and provide a framework for computing with objects of any finite type. Various research programs have been proposed in which higherorder objects, like functions on the real numbers, are represented/coded as real numbers, so as to make them amenable to the Turing framework. It is then a natural question whether there is any significant difference between the Kleene approach or the Turing-approachvia-codes. Continuous functions being well-studied in this context, we study functions of bounded variation, which have at most countably many points of discontinuity. A central result is the Jordan decomposition theorem that a function of bounded variation on $[0,1]$ equals the difference of two monotone functions. We show that for this theorem and related results, the difference between the Kleene approach and the Turing-approach-via-codes is huge, in that full second-order arithmetic readily comes to the fore in Kleene's approach, in the guise of Kleene's quantifier $\exists^{3}$.
\end{abstract}

\section{Introduction: Jordan, Turing, and Kleene}

In a nutshell, we study the computational properties of the Jordan decomposition theorem as in Theorem 1.1 and other results on functions of bounded variation, establishing the huge differences between the Turing and Kleene approaches to computability theory. For the rest of this section, we introduce the above italicised notions and sketch the contents of this paper in more detail. All technical notions are introduced in Section 2 while our main results are in Section 3 ,

First of all, Turing's famous 'machine' model, introduced in [45], constitutes the first intuitively convincing framework for computing with real numbers. Kleene's computation schemes S1-S9, introduced in [19]

\footnotetext{
Department of Mathematics, The University of Oslo, P.O. Box 1053, BLINDERN N-0316 OsLO, NorWAy

Department of Philosophy II, RUB Bochum, Germany

E-mail addresses: dnormann@math.uio.no, sasander@me.com.

Key words and phrases. Representations, computability theory, Kleene S1-S9, bounded variation.
} 
extend Turing's framework and provide a framework for computing with objects of any finite type. Now, various research programs have been proposed in which higher-order objects are represented/coded as real numbers or similar second-order representations, so as to make them amenable to the Turing framework. It is then a natural question whether there is any significant difference ${ }^{2}$ between the Kleene approach or the Turing-approach-via-codes. Continuous functions being well-studied ${ }^{2}$ in this context, we investigate functions of bounded variation, which have at most countably many points of discontinuity.

Secondly, the notion of bounded variation was first introduced by Jordan around 1881 ([18]) yielding a generalisation of Dirichlet's convergence theorems for Fourier series. Indeed, Dirichlet's convergence results are restricted to functions that are continuous except at a finite number of points, while functions of bounded variation can have (at most) countable many points of discontinuity, as also shown by Jordan, namely in [18, p. 230]. The fundamental theorem about functions of bounded variation is as follows and can be found in [18, p. 229].

Theorem 1.1 (Jordan decomposition theorem). A function $f:[0,1] \rightarrow$ $\mathbb{R}$ of bounded variation can be written as the difference of two nondecreasing functions $g, h:[0,1] \rightarrow \mathbb{R}$.

The computational properties of Theorem 1.1 have been studied extensively via second-order representations, namely in e.g. [14, 24, 29, 48]. The same holds for constructive analysis by [6, 17, 15, 39], involving different (but related) constructive enrichments. Now, finite iterations of the Turing jump suffice to compute $g, h$ from Theorem 1.1 in terms of represented functions $f$ of bounded variation by [24, Cor. 10].

Thirdly, in light of the previous, it is a natural question what the computational properties of Theorem 1.1 are in Kleene's framework. In particular, the following question is central to this paper.

How hard is it to compute (S1-S9) from $f:[0,1] \rightarrow \mathbb{R}$ of bounded variation, two monotone functions $g, h$ such that $f=g-h$ on $[0,1]$ ?

\footnotetext{
${ }^{1}$ Examples of such frameworks include: reverse mathematics (43, 44]), constructive analysis ([2, I.13], [3]), predicative analysis ([12]), and computable analysis (47]). Note that Bishop's constructive analysis is not based on Turing computability directly, but one of its 'intended models' is however (constructive) recursive mathematics, as discussed in [5]. One aim of Feferman's predicative analysis is to capture constructive reasoning in the sense of Bishop.

${ }^{2}$ The fan functional constitutes an early natural example of this difference: it has a computable code but is not S1-S9 computable (but S1-S9 computable in Kleene's $\exists^{2}$ from Section 2.2.1). The fan functional computes a modulus of uniform continuity for continuous functions on Cantor space; details may be found in [27.
} 
A functional that can perform this computational task will be called a Jordan realiser, introduced in Definition 3.2. A related and natural computational task is as follows.

How hard is it to compute $(S 1-S 9)$ from $f:[0,1] \rightarrow \mathbb{R}$ of bounded variation, the supremum $\sup _{x \in[0,1]} f(x)$ ?

A functional that can perform this computational task will be called a sup-realiser (see Definition 3.2). This task restricted to continuous functions is well-studied, and rather weak by [22, Footnote 6]. In light of the above, the following computational task is also natural:

How hard is it to compute $(S 1-S 9)$ from $f:[0,1] \rightarrow \mathbb{R}$ of bounded variation, a sequence $\left(x_{n}\right)_{n \in \mathbb{N}}$ listing the points of discontinuity of $f$ ? By way of a robustness result, we show that the above three tasks are the same modulo Kleene's $\exists^{2}$ from Section 2.2.1. Moreover, we show that Jordan realisers are hard to compute: no type two functional, in particular the functionals $S_{k}^{2}$ which decide $\Pi_{k}^{1}$-formulas (see Section 2.2.1), can compute a Jordan realiser. We also show that Jordan realisers are powerful: when combined with other natural functionals, one can go all the way up to Kleene's quantifier $\exists^{3}$ which yields full second-order arithmetic (see again Section 2.2.1 for the definition of $\left.\exists^{3}\right)$. We also study special cases of Jordan realisers, which connects to the computational tasks associated to the uncountability of $\mathbb{R}$.

Finally, our main results are obtained in Section 3 while some preliminary notions, including some essential parts of Kleene's higher-order computability theory, can be found in Section 2 .

\section{Preliminaries}

2.1. Kleene's higher-order computability theory. We first make our notion of 'computability' precise as follows.

(I) We adopt ZFC, i.e. Zermelo-Fraenkel set theory with the Axiom of Choice, as the official metatheory for all results, unless explicitly stated otherwise.

(II) We adopt Kleene's notion of higher-order computation as given by his nine clauses S1-S9 (see [27, Ch. 5] or [19]) as our official notion of 'computable'.

We refer to 27 for a thorough overview of higher-order computability theory. We do mention the distinction between 'normal' and 'nonnormal' functionals based on the following definition from [27, §5.4].

Definition 2.1. For $n \geq 2$, a functional of type $n$ is called normal if it computes Kleene's $\exists^{n}$ following S1-S9, and non-normal otherwise. 
We only make use of $\exists^{n}$ for $n=2,3$, as defined in Section 2.2.1.

It is a historical fact that higher-order computability theory, based on Kleene's S1-S9, has focused primarily on the world of normal functionals (see [27, §5.4] for this opinion). We have previous studied the computational properties of new non-normal functionals, namely those that compute the objects claimed to exist by:

- the Heine-Borel and Vitali covering theorems ([30,31, 33]),

- the Baire category theorem ([34]),

- local-global principles like Pincherle's theorem ([35]),

- the uncountability of $\mathbb{R}$ and the Bolzano-Weierstrass theorem for countable sets in Cantor space $([36,38])$,

- weak fragments of the Axiom of (countable) Choice ([37]).

In this paper, we continue this study for the Jordan decomposition theorem and other basic properties of functions of bounded variation. Next, we introduce some required higher-order notions in Section 2.2.

\subsection{Some higher-order notions.}

2.2.1. Some higher-order functionals. We introduce a number of comprehension functionals from the literature. We are dealing with conventional comprehension, i.e. only parameters over $\mathbb{N}$ and $\mathbb{N}^{\mathbb{N}}$ are allowed in formula classes like $\Pi_{k}^{1}$ et cetera.

First of all, the functional $\varphi$ as in $\left(\exists^{2}\right)$ is clearly discontinuous at $f=11 \ldots$; in fact, $\left(\exists^{2}\right)$ is equivalent to the existence of $F: \mathbb{R} \rightarrow \mathbb{R}$ such that $F(x)=1$ if $x>0$, and 0 otherwise (see [22, §3]).

$$
\left(\exists \varphi^{2} \leq_{2} 1\right)\left(\forall f^{1}\right)\left[\left(\exists n^{0}\right)(f(n)=0) \leftrightarrow \varphi(f)=0\right] .
$$

Intuitively speaking, the functional $\varphi$ from $\left(\exists^{2}\right)$ can decide the truth of any $\Sigma_{1}^{0}$-formula in its (Kleene) normal form. Related to $\left(\exists^{2}\right)$, the functional $\mu^{2}$ in $\left(\mu^{2}\right)$ is also called Feferman's $\mu$ ([1]), defined as follows:

$$
\begin{aligned}
\left(\exists \mu^{2}\right)\left(\forall f^{1}\right)[(\exists n)(f(n)=0) \rightarrow[ & f(\mu(f))=0 \wedge(\forall i<\mu(f))(f(i) \neq 0)] \\
& \wedge[(\forall n)(f(n) \neq 0) \rightarrow \mu(f)=0]] . \quad\left(\mu^{2}\right)
\end{aligned}
$$

We have $\left(\exists^{2}\right) \leftrightarrow\left(\mu^{2}\right)$ over a weak system by [21, Prop. 3.4 and Cor. 3.5]) while $\mu^{2}$ is readily computed from $\varphi^{2}$ in $\left(\exists^{2}\right)$. The third-order functional from $\left(\exists^{2}\right)$ is also called 'Kleene's quantifier $\exists^{2}$ ', and we use the same convention for other functionals.

Secondly, $\mathrm{S}^{2}$ as in $\left(\mathrm{S}^{2}\right)$ is called the Suslin functional ([1,22]):

$$
\left(\exists \mathrm{S}^{2} \leq_{2} 1\right)\left(\forall f^{1}\right)\left[\left(\exists g^{1}\right)\left(\forall n^{0}\right)(f(\bar{g} n)=0) \leftrightarrow \mathrm{S}(f)=0\right] .
$$


Intuitively, the Suslin functional $S^{2}$ can decide the truth of any $\Sigma_{1}^{1}$ formula in its normal form. We similarly define the functional $S_{k}^{2}$ which decides the truth or falsity of $\Sigma_{k}^{1}$-formulas (again in normal form). We note that the operators $\nu_{n}$ from [10, p. 129] are essentially $\mathrm{S}_{n}^{2}$ strengthened to return a witness to the $\Sigma_{n}^{1}$-formula at hand. As suggested by its name, $\nu_{k}$ is the restriction of Hilbert-Bernays' $\nu$ from [16, p. 495] to $\Sigma_{k}^{1}$-formulas. We sometimes use $\mathrm{S}_{0}^{2}$ and $\mathrm{S}_{1}^{2}$ to denote $\exists^{2}$ and $\mathrm{S}^{2}$.

Thirdly, second-order arithmetic is readily derived from the following:

$$
\left(\exists E^{3} \leq_{3} 1\right)\left(\forall Y^{2}\right)\left[\left(\exists f^{1}\right)(Y(f)=0) \leftrightarrow E(Y)=0\right] .
$$

The functional from $\left(\exists^{3}\right)$ is also called 'Kleene's quantifier $\exists^{3}$ '. HilbertBernays' $\nu$ from [16, p. 495] trivially computes $\exists^{3}$.

Finally, the functionals $S_{k}^{2}$ are defined using the usual formula class $\Pi_{k}^{1}$, i.e. only allowing first- and second-order parameters. We have dubbed this the conventional approach and the associated functionals are captured by the umbrella term conventional comprehension. Comprehension involving third-order parameters has previously (only) been studied in [12,20], to the best of our knowledge.

2.2.2. Some higher-order definitions. We introduce some required definitions, which are all standard.

First of all, a fruitful and faithful approach is the representation of sets by characteristic functions (see e.g. [25, 33, 34, 37, 40 42]), wellknown from e.g. measure and probability theory. We shall use this approach, always assuming $\exists^{2}$ to make sure open sets represented by countable unions of basic opens are indeed sets in our sense.

Secondly, we now turn to countable sets. Of course, the notion of 'countable set' can be formalised in various ways, as follows.

Definition 2.2. [Enumerable set] A set $A \subset \mathbb{R}$ is enumerable if there is a sequence $\left(x_{n}\right)_{n \in \mathbb{N}}$ such that $(\forall x \in \mathbb{R})\left(x \in A \leftrightarrow(\exists n \in \mathbb{N})\left(x=x_{n}\right)\right)$.

Definition 2.2 reflects the notion of 'countable set' from reverse mathematics ([43, V.4.2]). Our definition of 'countable set' is as follows.

Definition 2.3. [Countable set] A set $A \subset \mathbb{R}$ is countable if there is $Y: \mathbb{R} \rightarrow \mathbb{N}$ such that

$$
(\forall x, y \in A)\left(Y(x)={ }_{0} Y(y) \rightarrow x=y\right) .
$$

The functional $Y$ as in (2.1) is called injective on $A$ or an injection on $A$. If $Y: \mathbb{R} \rightarrow \mathbb{N}$ is also surjective, i.e. $(\forall n \in \mathbb{N})(\exists x \in A)(Y(x)=n)$, we call $A$ strongly countable. The functional $Y$ is then called bijective on $A$ or a bijection on $A$. 
The first part of Definition 2.3 is from Kunen's set theory textbook ([26, p. 63]) and the second part is taken from Hrbacek-Jech's set theory textbook [17] (where the term 'countable' is used instead of 'strongly countable'). According to Veldman ([46, p. 292]), Brouwer studies set theory based on injections in [9]. Hereonafter, 'strongly countable' and 'countable' shall exclusively refer to Definition 2.3.

\section{MAin Results}

In this section, we shall obtain our main results as follows. Recall that a Jordan realiser outputs the monotone functions claimed to exist by the Jordan decomposition theorem as in Theorem 1.1.

- We introduce Jordan realisers and other functionals witnessing basic properties of functions of bounded variation; we show that three of these are computationally equivalent (Section 3.1).

- We show that Jordan realisers are hard to compute based on results from 36] (Section 3.2).

- We show that Kleene's $\exists^{3}$ can be computed from $\exists^{2}$, a Jordan realiser, and a well-ordering of [0,1] (Section 3.3).

- We show that Jordan realisers remain hard to compute even if we severely restrict the output (Section 3.4).

3.1. Jordan realisers and equivalent formulations. We introduce functionals witnessing basic properties of functions of bounded variation, including the Jordan decomposition theorem (Theorem 1.1). We show that three of these are computationally equivalent given $\exists^{2}$.

As noted above, we always assume $\exists^{2}$ but specify the use when essential. This means that we can use the concept of Kleene-computability over $\mathbb{R}$ or $[0,1]$ without focusing on how these spaces are represented.

First of all, as to definitions, the total variation of a function $f$ : $[a, b] \rightarrow \mathbb{R}$ is (nowadays) defined as follows:

$$
V_{a}^{b}(f):=\sup _{a \leq x_{0}<\cdots<x_{n} \leq b} \sum_{i=0}^{n}\left|f\left(x_{i}\right)-f\left(x_{i+1}\right)\right| .
$$

If this quantity exists and is finite, one says that $f$ has bounded variation on $[a, b]$. Now, the notion of bounded variation is defined in [29] without mentioning the supremum in (3.1); this approach can also be found in [6, 7, 24]. Hence, we shall hereafter distinguish between the following two notions. As it happens, Jordan seems to use item (国) of Definition 3.1 in [18, p. 228-229], providing further motivation for the functionals introduced in Definition 3.2 .

Definition 3.1. [Variations on variation] 
(a) The function $f:[a, b] \rightarrow \mathbb{R}$ has bounded variation on $[a, b]$ if there is $k_{0} \in \mathbb{N}$ such that $k_{0} \geq \sum_{i=0}^{n}\left|f\left(x_{i}\right)-f\left(x_{i+1}\right)\right|$ for any partition $x_{0}=a<x_{1}<\cdots<x_{n-1}<x_{n}=b$.

(b) The function $f:[a, b] \rightarrow \mathbb{R}$ has a variation on $[a, b]$ if the supremum in (3.1) exists and is finite.

We can now introduce the following notion of 'realiser' for the Jordan decomposition theorem and related functionals.

\section{Definition 3.2.}

- A Jordan realiser is a partial functional $\mathcal{J}$ of type 3 taking as input a function $f:[0,1] \rightarrow \mathbb{R}$ which has bounded variation (item (国) in Definition 3.1), and providing a pair $(g, h)$ of increasing functions $g$ and $h$ such that $f=g-h$ on $[0,1]$.

- A weak Jordan realiser is a partial functional $\mathcal{J}_{\mathrm{w}}$ of type 3 taking as inputs a function $f:[0,1] \rightarrow \mathbb{R}$ and its bounded variation $V_{0}^{1}(f)$ (item (b) in Definition [3.1), and providing a pair $(g, h)$ of increasing functions $g$ and $h$ such that $f=g-h$ on $[0,1]$.

- A sup-realiser is a partial functional $\mathcal{S}$ of type 3 taking as input a function $f:[0,1] \rightarrow \mathbb{R}$ which has bounded variation (item (a) in Definition 3.1), and providing the supremum $\sup _{x \in[0,1]} f(x)$.

- A continuity-realiser is a partial functional $\mathcal{L}$ of type 3 taking as input a function $f:[0,1] \rightarrow \mathbb{R}$ which has bounded variation (item (a) in Definition 3.1), and providing a sequence $\left(x_{n}\right)_{n \in \mathbb{N}}$ which lists all points of discontinuity of $f$ on $[0,1]$.

Next, we need the following lemma. The use of $\exists^{2}$ is perhaps superfluous in light of the constructive proof in [8], but the latter seems to make essential use of the Axiom of (countable) Choice.

Lemma 3.3. There is a functional $\mathcal{D}$, computable in $\exists^{2}$, such that if $f:[0,1] \rightarrow \mathbb{R}$ is increasing (decreasing), then $\mathcal{D}(f)$ enumerates all points of discontinuity of $f$ on $[0,1]$.

Proof. Let $\left\{q_{i}\right\}_{i \in \mathbb{N}}$ be an enumeration of $\mathbb{Q} \cap[0,1]$, let $f:[0,1] \rightarrow \mathbb{R}$ be monotone, and define $a_{i}:=f\left(q_{i}\right)$. Let $A$ be the set of pairs of rationals $p<r$ such that there is no $i$ with $p<a_{i}<r$. For $(p, r) \in A$, define

$$
x_{p, r}:=\sup \left\{q_{i}: a_{i} \leq p\right\}=\inf \left\{q_{j}: r \leq a_{j}\right\} .
$$

It is easy to see that the reals in (3.2) are equal; indeed, the existence of a rational $q_{i}$ between them, together with assuming that $(p, r) \in A$, leads to a contradiction.

Then all discontinuities of $f$ will be among the elements $x_{p, r}$ for $(p, r) \in A$. Clearly, $i \mapsto a_{i}, A$ and $(p, r) \mapsto x_{p, r}$ for $(p, r) \in A$ are computable in $f$ and $\exists^{2}$. This ends the proof. 
Finally, we show that three 'non-weak' realisers from Definition 3.2 are in fact one and the same, in part based on Lemma 3.3.

Theorem 3.4. Assuming $\exists^{2}$, Jordan realisers, sup-realisers, and continuity realisers are computationally equivalent.

Proof. We first show that a continuity realiser computes a sup-realiser. To this end, let $f$ be of bounded variation on $[0,1]$ and let $\mathcal{L}(f)=$ $\left(x_{n}\right)_{n \in \mathbb{N}}$ be a list of its points of discontinuity. From $\mathcal{L}(f)$ we can find a list $\left(y_{j}\right)_{j \in \mathbb{N}}$ containing both the points of discontinuity and all rational numbers in $[0,1]$. Then we can compute

$$
\mathcal{S}(f)=\sup \{f(x): x \in[0,1]\}=\sup \left\{f\left(y_{j}\right): j \in \mathbb{N}\right\},
$$

since $\sup \left\{f\left(y_{j}\right): j \in \mathbb{N}\right\}$ is computable from $\mathcal{L}(f), f$, and $\exists^{2}$.

Secondly, that a Jordan realiser computes a continuity realiser, assuming $\exists^{2}$, is immediate from Lemma 3.3 .

Thirdly, we show that a sup-realiser $\mathcal{S}$ computes a Jordan realiser. To this end, let $a$ and $b$ be such that $0 \leq a<b \leq 1$ and define

$$
\mathcal{S}_{a, b}^{+}(f):=\sup _{x \in[a, b]} f(x) \text { and } \mathcal{S}_{a, b}^{-}(f):=\inf _{x \in[a, b]} f(x) .
$$

These functionals are clearly computable in $\mathcal{S}$, for $f:[0,1] \rightarrow \mathbb{R}$ a function of bounded variation. Now let $\operatorname{Var}(P, f)$ be the $\operatorname{sum} \sum_{i=0}^{n-1} \mid f\left(x_{i+1}\right)-$ $f\left(x_{i}\right) \mid$ for a partition $P=\left\{0=x_{0}<\cdots<x_{n}=1\right\}$ of $[0,1]$, while $\operatorname{Var}^{+}(P, f)$ is the sum of all positive differences $f\left(x_{i+1}\right)-f\left(x_{i}\right)$.

Claim 3.5. To compute a Jordan realiser, it suffices to compute $\Delta(f):=$ $\sup _{P} \operatorname{Var}^{+}(P, f)$, where $P$ varies over all partitions of $[0,1]$.

To prove Claim [3.5, we compute increasing functions $f^{+}$and $f^{-}$ such that $f=f^{+}-f^{-}$. Without loss of generality, we may assume that $f(0)=0$, and by symmetry it suffices to compute $f^{+}$. We can define $f^{+}(0)=0$ and $f^{+}(x)=\Delta\left(f_{x}\right)$ for $x>0$, where $f_{x}(y)=f\left(\frac{y}{x}\right)$. This ends the proof of Claim 3.5.

We now employ the functionals $\mathcal{S}_{a, b}^{+}$and $\mathcal{S}_{a, b}^{-}$from (3.4) as follows.

Definition 3.6. Let $f$ be of bounded variation on $[0,1]$ and let $n \in \mathbb{N}$. An $n, f$-trail is a sequence $k_{0}, \ldots, k_{2 m-1}$ such that $0 \leq k_{0}<\ldots<$ $k_{2 m-1}<n$ and such that when $0 \leq j<m$ we have

$$
\mathcal{S}_{\frac{k_{2 j}}{n}, \frac{k_{2 j}+1}{n}}^{-}(f)<\mathcal{S}_{\frac{k_{2 j+1}}{n}}^{+}, \frac{k_{2 j+1}+1}{n}(f) .
$$

Define the positive $n$-move $\bar{M}(n, f)$ as the maximal value of

$$
\sum_{j=0}^{m-1}\left(\mathcal{S}_{\frac{k_{2 j+1}}{n}, \frac{k_{2 j+1}+1}{n}}^{+}(f)-\mathcal{S}_{\frac{k_{2 j}}{n}, \frac{k_{2 j}+1}{n}}^{-}(f)\right)
$$


where $k_{0}, \ldots, k_{2 m-1}$ varies over all $n, f$-trails.

Claim 3.7. For each $n \in \mathbb{N}$, we have that $\bar{M}(n, f) \leq \Delta(f)$.

To prove Claim [3.7, it suffices to show that $\bar{M}(n, f) \leq \Delta(f)+\epsilon$ for each $\epsilon>0$. Let $k_{0}, \ldots, k_{2 m-1}$ be an $n, f$-trail giving the value of $\bar{M}(n, f)$. For each $j<m$, select $x_{j} \in\left[\frac{k_{2 j+1}}{n}, \frac{k_{2 j+1}+1}{n}\right]$ such that $f\left(x_{k}\right)>\mathcal{S}_{\frac{k_{2 j+1}}{n}, \frac{k_{2 j+1}+1}{n}}^{+}(f)-\frac{\epsilon}{2 m}$ and $y_{j} \in\left[\frac{k_{2 j}}{n}, \frac{k_{2 j}+1}{n}\right]$ such that $f\left(y_{k}\right)<$ $\mathcal{S}_{\frac{k_{2 j}}{n}, \frac{k_{2 j}+1}{n}}^{-}(f)^{n}+\frac{\epsilon}{2 m}$. For any partition $P$ containing all points $x_{j}$ and $y_{j}$ we have that $\operatorname{Var}^{+}(P, f)>\bar{M}(n, f)-\epsilon$. As a result, we obtain

$$
\bar{M}(n, f)<\operatorname{Var}^{+}(P, f)+\epsilon \leq \Delta(f)+\epsilon,
$$

which ends the proof of Claim 3.7 .

Claim 3.8. Let $f$ be of bounded variation on $[0,1]$ and let $P$ be a partition of $[0,1]$. Then there is $n \in \mathbb{N}$ such that $\operatorname{Var}^{+}(P, f) \leq \bar{M}(n, f)$.

To prove Claim 3.8, fix $P=\left\{0 \leq s_{0}<\cdots<s_{m^{\prime}} \leq 1\right\}$. Without loss of generality, we may assume that $f\left(s_{0}\right)<f\left(s_{1}\right)>f\left(s_{2}\right)<\cdots<$ $f\left(s_{m^{\prime}}\right)$, i.e. the values $f\left(s_{i}\right)$ alternate between going up and going down, so $m^{\prime}$ is an uneven number $2 m-1$. Let $n$ be such that each $\left[\frac{k}{n}, \frac{k+1}{n}\right]$, for $k<n$, contains at most one $s_{i}$ and each $s_{i}$ is contained in exactly one $\left[\frac{k_{i}}{n}, \frac{k_{i}+1}{n}\right]$. Then $k_{0}, \ldots, k_{2 m-1}$ is an $n, f$-trail witnessing that $\operatorname{Var}^{+}(P, f) \leq \bar{M}(n, f)$. This ends the proof of Claim 3.8 .

Finally, by the above three claims, we have $\Delta(f)=\sup _{n \in \mathbb{N}} \bar{M}(n, f)$. Since we can compute the latter from $\mathcal{S}$ and $\exists^{2}$, the former is likewise computable. As we can compute a Jordan realiser from $\Delta$, the proof of Theorem 3.4 is complete.

In conclusion, as perhaps expected in light of (3.3), rather effective and pointwise approximation results exist for functions of bounded variation at points of continuity (see e.g. [11, p. 261]). For points of discontinuity, it seems one only approximates the average of the left and right limits, i.e. not the function value itself.

3.2. Jordan realiser and countable sets. We show that Jordan realisers are hard to compute by connecting them to computability theoretic results pertaining to countable sets from [36]. Recall the definitions from Section 2.2 .2 pertaining to the latter notion.

Now, the most fundamental property of countable sets is that they can be enumerated, i.e. listed as a sequence, as explicitly noted by e.g. Borel in [4] in his early discussions of the Heine-Borel theorem. Next, 
we show that Jordan realisers can indeed enumerate countable sets as in Definition 2.3.

Theorem 3.9. Together with $\exists^{2}$, a Jordan realiser $\mathcal{J}$ can perform the following computational procedures.

- Given a set $A \subset[0,1]$ and $Y:[0,1] \rightarrow \mathbb{N}$ injective on $A$, produce a sequence $\left(x_{n}\right)_{n \in \mathbb{N}}$ listing exactly the elements of $A$.

- Given $F:[0,1] \rightarrow[0,1], A \subset[0,1]$, and $Y:[0,1] \rightarrow \mathbb{N}$ injective on $A$, produce $\sup _{x \in A} F(x)$.

Proof. We only need to establish the first item, as the second item readily follows from the first one using $\exists^{2}$. Let $A \subset[0,1]$ be countable, i.e. there is $Y:[0,1] \rightarrow \mathbb{N}$ which is injective on $A$. Use $\exists^{2}$ to define the function $f: \mathbb{R} \rightarrow \mathbb{R}$ defined as follows:

$$
f(x):=\left\{\begin{array}{ll}
\frac{1}{2^{Y(x)+1}} & x \in A \\
0 & \text { otherwise }
\end{array} .\right.
$$

Following item (国) in Definition 3.1, the function $f$ has bounded variation on $[0,1]$ as any sum $\sum_{i=0}^{n}\left|f\left(x_{i}\right)-f\left(x_{i+1}\right)\right|$ is at most $\sum_{i=0}^{n} \frac{1}{2^{i+1}}$ for $x_{i}$ in $[0,1]$ and $i \leq n+1$. Now let $\mathcal{J}(f)=(g, h)$ be such that $f=g-h$ on $[0,1]$ and recall $\mathcal{D}$ from Lemma 3.3 . Use $\exists^{2}$ to define the sequence $\left(x_{n}\right)_{n \in \mathbb{N}}$ as all the reals in $\mathcal{D}(g)$ and $\mathcal{D}(h)$. Now consider the following formula for any $x \in[0,1]$ :

$$
\left[(\exists n \in \mathbb{N})\left(x=x_{n}\right) \wedge g(x) \neq h(x)\right] \leftrightarrow x \in A .
$$

The forward direction in (3.6) is immediate as $g(x) \neq h(x)$ for $x \in[0,1]$ implies $f(x)>0$, and hence $x \in A$ by definition. For the reverse direction, fix $x \in A$ and note that $0<f(x)=g(x)-h(x)$ by the definition of $f$ in (3.6), i.e. $g(x) \neq h(x)$ holds. Moreover, in case $(\forall n \in \mathbb{N})\left(x \neq x_{n}\right)$, then $g$ and $h$ are continuous at $x$, by the definition of $\left(x_{n}\right)_{n \in \mathbb{N}}$. Hence, $f$ is continuous at $x$, which is only possible if $f(x)=0$, but the latter implies $x \notin A$, by (3.5), a contradiction. In this way, we obtain (3.6) and we may enumerate $A$ by removing from $\left(x_{n}\right)_{n \in \mathbb{N}}$ all elements not in $A$, which can be done using $\exists^{2}$.

Secondly, weak Jordan realisers can enumerate strongly countable sets (Definition 2.3).

Corollary 3.10. Together with $\exists^{2}$, a weak Jordan realiser $\mathcal{J}_{\mathrm{w}}$ can perform the following computational procedures.

- Given a set $A \subset[0,1]$ and $Y:[0,1] \rightarrow \mathbb{N}$ bijective on $A$, produce a sequence $\left(x_{n}\right)_{n \in \mathbb{N}}$ listing exactly the elements of $A$.

- Given $F:[0,1] \rightarrow[0,1], A \subset[0,1]$, and $Y:[0,1] \rightarrow \mathbb{N}$ bijective on $A$, produce $\sup _{x \in A} F(x)$. 
Proof. Following item (b) in Definition 3.1, the function $f$ in (3.5) has total variation exactly 1 in case $Y$ is additionally a bijection.

A weak Jordan realiser cannot compute a Jordan realiser; this remains true if we combine the former with an arbitrary type 2 functional. Since the proof of this claim is rather lengthy, we have omitted the former from this paper.

Thirdly, the functional $\Omega_{\mathrm{BW}}$ introduced and studied in [36, §4], performs the computational procedure from the second item in Theorem 3.9 , leading to the following corollary.

Corollary 3.11. Together with the Suslin functional $\mathrm{S}^{2}$, a Jordan realiser $\mathcal{J}$ computes $\mathrm{S}_{2}^{2}$, i.e. a realiser for $\Pi_{2}^{1}-\mathrm{CA}_{0}$.

Proof. By [36, Theorem 4.6.(b)], $\Omega_{\mathrm{BW}}+\mathrm{S}^{2}$ computes $\mathrm{S}_{2}^{2}$, while a Jordan realiser $\mathcal{J}$ computes $\Omega_{\mathrm{BW}}$ by Theorem 3.9 .

Finally, under the additional set-theoretic hypothesis $\mathrm{V}=\mathrm{L}$, the combination $\Omega_{\mathrm{BW}}+\mathrm{S}^{2}$ even computes $\exists^{3}$ by $[36$, Theorem 4.6.(c)]. An obvious question is whether a similar result can be obtained within ZFC, which is the topic of the following section.

3.3. Computing Kleene's $\exists^{3}$ from Jordan realisers. We show that Kleene's quantifier $\exists^{3}$ is computable in the combination of:

- Kleene's quantifier $\exists^{2}$,

- any Jordan realiser $\mathcal{J}$ (or: $\Omega_{\mathrm{BW}}$ from Section 3.2),

- a well-ordering $\prec$ of $[0,1]$.

We note that the third item exists by the Axiom of Choice. Assuming $\prec$ and $\preceq$ are the irreflexive and reflexive versions of the same wellordering of $[0,1]$, they are computable in each other and $\exists^{2}$.

Theorem 3.12. Let $\mathcal{J}$ be a Jordan realiser. Then $\exists^{3}$ is Kleenecomputable in $\mathcal{J}, \prec$, and $\exists^{2}$.

Proof. We actually prove a slightly stronger result. Let $\Omega$ be a partial functional of type 3 such that $\Omega(X)$ terminates whenever $X \subset \mathbb{R}$ has at most one element, and $\Omega(X) \in X$ whenever $X$ contains exactly one element. One readily shows that $\Omega$ is computationally equivalent to $\Omega_{\mathrm{BW}}$, given $\exists^{2}$. We now show that $\exists^{3}$ is computable in $\Omega$, $\prec$, and $\exists^{2}$.

\footnotetext{
${ }^{3}$ To obtain an enumeration of $A \subset[0,1]$ given $Y:[0,1] \rightarrow \mathbb{R}$ injective on $A$, define $E_{n}:=\{x \in A: Y(x)=n\}$ and define $x_{n}:=\Omega\left(E_{n}\right)$ in case the latter is in $E_{n}$, and 0 otherwise.
} 
We let $x, y$ vary over $[0,1]$ and we fix $h:[0,1] \rightarrow\{0,1\}$. We aim to compute $\exists^{3}(h)$ by deciding the truth of the formula $(\exists x \in[0,1])(h(x)=$ 1). To this end, consider the functionals $E_{\prec x}$ and $E_{\preceq x}$ defined as:

$E_{\prec x}(h)=1 \leftrightarrow(\exists y \prec x)(h(y)=1)$ and $E_{\preceq x}(h)=1 \leftrightarrow(\exists y \preceq x)(h(y)=1)$.

We shall show that these are computable in $\Omega$ and $\exists^{2}$, uniformly in $x$. Note that $E_{\preceq x}$ is trivially computable in $E_{\prec x}$, uniformly in $x$, so we settle for computing $E_{\prec x}(h)$. The argument will be by the recursion theorem, so we give the algorithm for computing $E_{\prec x}(h)$ using $x, h, \Omega$ and $E_{\prec y}$ for $y \prec x$. Now let $x$ and $h$ be fixed and define $h_{x}$ as:

$$
h_{x}(y):= \begin{cases}0 & \text { if } x \preceq y \\ h(y) & \text { if } y \prec x \wedge(\forall z \prec y)(h(z)=0) \\ 0 & \text { otherwise }\end{cases}
$$

where we use $E_{\prec y}$ to decide whether the second case holds. Then $h_{x}$ is constant zero if $(\forall y \prec x)(h(y)=0)$, and if not, $h_{x}$ takes the value 1 in exactly the least point $y \prec x$ where $h(y)=1$. Hence, we have

$$
(\exists y \prec x)(h(y)=1) \leftrightarrow\left[\Omega\left(h_{x}\right) \prec x \wedge h\left(\Omega\left(h_{x}\right)\right)=1\right] .
$$

We now apply the recursion theorem to find an index $e \in \mathbb{N}$ such that for all $x \in[0,1]$ and $h:[0,1] \rightarrow\{0,1\}$ (and well-orderings $\preceq$ ), we have

$$
\{e\}(\Omega, h, x, \preceq) \simeq E_{\preceq x}(h) .
$$

Then we use transfinite induction over $\preceq$ to prove that $e \in \mathbb{N}$ defines a total functional doing what it is supposed to do. For readers not familiar with this use of the recursion theorem, what we do is defining $\left\{e_{0}\right\}(d, \Omega, h, x, \preceq)$ as in the construction, but replacing all uses of $E_{\preceq y}\left(h^{\prime}\right)$ by $\{d\}\left(\Omega, h^{\prime}, y, \preceq\right)$; we then use the fact that there is an index $e$ such that $\{e\}(\ldots) \simeq\left\{e_{0}\right\}(e, \ldots)$, where '...' are the other parameters.

Having established the computability of each $E_{\prec x}$ from $x$, we can use the same trick to compute $\exists^{3}(h)$ as follows: construct from $h$ a function $h^{\prime}$ that takes the value 1 in at most one place, namely the $\prec$-least $x$ where $h(x)=1$, in case such exists.

Finally, we note that Kleene's computation scheme S9 is essentially a 'hard-coded' version of the recursion theorem for S1-S9, while S1S8 merely define (higher-order) primitive recursion. In this way, the recursion theorem is central to $\mathrm{S} 1-\mathrm{S} 9$, although we have previously witnessed S1-S9 computations via primitive recursive terms. 
3.4. Jordan realisers and the uncountability of $\mathbb{R}$. We show that a number of interesting functionals, including 'heavily restricted' Jordan realisers, are (still) quite hard to compute, based on the computational properties of the uncountability of $\mathbb{R}$ pioneered in [36].

First of all, in more detail, Theorem 3.4 implies that a Jordan realiser can enumerate all points of discontinuity of a function of bounded variation. It is then a natural question whether Jordan realisers remain hard to compute if we only require the output to be e.g. one point of continuity. By way of an answer, Theorem 3.15 lists a number of interesting functionals -including the aforementioned 'one-point' Jordan realisers- that compute functionals witnessing the uncountability of $\mathbb{R}$. Functionals related to the uncountability of $\mathbb{R}$ are special in the following sense.

By the previous sections, Jordan realisers have surprising properties and are a nice addition to the pantheon of interesting non-normal functionals stemming from ordinary mathematics (see [30 38] or Section 2.1 for other examples). It is then a natural question what the weakest such functional is; a candidate is provided by the uncountability of $\mathbb{R}$, which can be formulated in various guises as follows.

- Cantor's theorem: there is no surjection from $\mathbb{N}$ to $\mathbb{R}$.

- NIN: there is no injection from $[0,1]$ to $\mathbb{N}$.

- NBI: there is no bijection from $[0,1]$ to $\mathbb{N}$.

Cantor's theorem is provable in constructive and computable mathematics ([3,43]), while there is even an efficient algorithm to compute from a sequence of reals, a real not in that sequence ([13]). As explored in [36], NIN and NBI are hard to prove in terms of conventional comprehension. We will not study NBI in this paper while Cantor's theorem and NIN give rise to the following specifications.

Definition 3.13. [Realisers for the uncountability of $\mathbb{R}$ ]

- A Cantor functional/realiser takes as input $A \subset[0,1]$ and $Y$ : $[0,1] \rightarrow \mathbb{N}$ such that $Y$ is injective on $A$, and outputs $x \notin A$.

- A weak Cantor realiser takes as input $A \subset[0,1]$ and $Y$ : $[0,1] \rightarrow \mathbb{N}$ such that $Y$ is bijective on $A$, and outputs $x \notin A$.

- A NIN-realiser takes as input $Y:[0,1] \rightarrow \mathbb{N}$ and outputs $x, y \in$ $[0,1]$ with $x \neq y \wedge Y(x)=Y(y)$.

As explored in [36], NIN-realisers are among the weakest non-normal functionals originating from ordinary mathematics we have studied. 
Moreover, one readily proves that a NIN-realiser computes a Cantor realiser, while the latter are still hard to compute as follows.

Theorem 3.14. No type 2 functional computes a weak Cantor realiser.

Proof. Fix some functional $F^{2}$ and assume wlog that $F$ computes $\exists^{2}$. Assume there is a Cantor realiser $\mathcal{C}$ computable in $F$. Now let $A$ be the set of reals computable in $F$ and define $Y:[0,1] \rightarrow \mathbb{N}$ as follows using Gandy selection (see [27, 37] for an introduction): for the first part, define $Y(x)$ as an index for computing $x$ from $F$ in case $x \in A$; we put $Y(x):=0$ in case $x \notin A$. By assumption, $\mathcal{C}(A, Y)$ terminates as $Y$ is injective on $A$. Since the restriction of $Y$ to $A$ is partially computable in $F$, all oracle calls of the form ' $x \in A$ ' will be answered with yes, since $x$ then is computable in $F$. Hence, all oracle calls for the value $Y(x)$ can be answered computably in $F$. In this way, $\mathcal{C}(A, Y)$ is computable in $F$, which also follows from [36, Lemma 2.15]. Hence, $\mathcal{C}(A, Y) \in A$ by definition, a contradiction. The proof remains valid if we extend $A$ to some $B \subset[0,1]$ and extend $Y$ to $Z:[0,1] \rightarrow \mathbb{N}$ bijective on $B$.

Secondly, it is fairly trivial to prove (classically) that there is no continuous injection from $[0,1]$ to $\mathbb{Q}$, based on the intermediate value theorem. Now consider the following principle, which expresses a very special case of the uncountability of $\mathbb{R}$.

- $N I N_{B V}$ : there is no injection from $[0,1]$ to $\mathbb{Q}$ that has bounded variation (item (国) in Definition 3.1).

One readily establishes the equivalence NIN $\leftrightarrow$ NIN $_{\text {BV }}$ over a weak system, following the proof of Theorem 3.15. By the latter and Theorem 3.14, while NIN-realisers are defined for all $Y:[0,1] \rightarrow \mathbb{N}$, the restriction to functions of bounded variation, which only have countably many points of discontinuity, is (still) hard to compute and intermediate between Cantor and NIN-realisers.

Thirdly, we have the following theorem where the functional

$$
\mathfrak{L}(f)(s):=\int_{0}^{+\infty} e^{-s t} f(t) d t
$$

is the Laplace transform of $f: \mathbb{R} \rightarrow \mathbb{R}$. Since we restrict to functions of bounded variation, we interpret $\mathfrak{L}(f)$ as the limit of Riemann integrals, if the latter exists. It is well-known that if $\mathfrak{L}(f)$ and $\mathfrak{L}(g)$ exists and are equal everywhere, $f$ and $g$ are equal almost everywhere, inspiring the final -considerably weaker- item in Theorem 3.15. In the below items, 'bounded variation' refers to item (国) of Definition 3.1 .

\footnotetext{
${ }^{4}$ Let $N$ be a NIN-realiser and let $A \subset[0,1]$ and $Y:[0,1] \rightarrow \mathbb{N}$ be such that $Y$ is injective on $A$. Define $Z:[0,1] \rightarrow \mathbb{N}$ as follows: $Z(x):=Y(x)+1$ in case $x \in A$, and 0 otherwise. Clearly, $N(Z)(1) \notin A$ as required for a Cantor functonal.
} 
Theorem 3.15. Assuming $\exists^{2}$, a Cantor realiser can be computed from a functional performing any of the following tasks.

- For $f:[0,1] \rightarrow \mathbb{Q}$ which has bounded variation, find $x, y \in[0,1]$ such that $x \neq y$ and $f(x)=f(y)$.

- For $f:[0,1] \rightarrow \mathbb{R}$ which has bounded variation, find a point of continuity in $[0,1]$.

- If $f:[0,1] \rightarrow[0,1]$ is Riemann integrable (or has bounded variation) with $\int_{0}^{1} f(x) d x=0$, find $y \in[0,1]$ with $f(y)=0$.

- If $f, g: \mathbb{R} \rightarrow \mathbb{R}$ satisfy the following:

$-f, g$ have bounded variation on $[0, a]$ for any $a \in \mathbb{R}^{+}$,

- $\mathfrak{L}(f)$ and $\mathfrak{L}(g)$ exists and are equal on $[0,+\infty)$,

find $x \in(0, \infty)$ with $f(x)=g(x)$.

Any NIN-realiser computes a functional as in the first item. Assuming $\exists^{2}$, a weak Cantor realiser can be computed from a functional performing any of the above tasks restricted as in item (b) of Definition [3.1.

Proof. The penultimate sentence is immediate as $\mathbb{Q}$ and $\mathbb{N}$ are bijective. Now fix some countable set $A \subset[0,1]$ and $Y:[0,1] \rightarrow \mathbb{N}$ injective on $A$. Consider $f:[0,1] \rightarrow \mathbb{R}$ as in (3.5) and recall it has bounded variation. For the second item, if $x_{0} \in[0,1]$ is a point of continuity of $f$, we must have $f\left(x_{0}\right)=0$ by continuity. Then $x_{0} \notin A$ by definition, as required. For the first item, in case $x \neq y$ and $f(x)=f(y)$, we must have $x \notin A$ or $y \notin A$, in light of $(3.5)$.

For the third item, consider $f:[0,1] \rightarrow \mathbb{R}$ as in (3.5); that $\int_{0}^{1} f(x) d x$ exists and equals 0 , follows from the usual $\varepsilon$ - $\delta$-definition of Riemann integrability. Indeed, fix $\varepsilon_{0}>0$ and find $k_{0} \in \mathbb{N}$ such that $\frac{1}{2^{k_{0}}}<\varepsilon_{0}$. Let $P$ be a partition $x_{0}:=0, x_{1}, \ldots, x_{n}, x_{n+1}:=1$ of $[0,1]$ with $t_{i} \in\left[x_{i}, x_{i+1}\right]$ for $i \leq n$ and with mesh $\|P\|:=\max _{i \leq n}\left(x_{i+1}-x_{i}\right)$ at most $\frac{1}{2^{k_{0}}}$. Then the Riemann sum $S(f, P):=\sum_{i \leq n} f\left(t_{i}\right)\left(x_{i+1}-x_{i}\right)$ satisfies

$$
S(f, P) \leq \frac{1}{2^{k_{0}}} \sum_{i \leq n} f\left(t_{i}\right) \leq \frac{1}{2^{k_{0}}} \sum_{i \leq n} \frac{1}{2^{i+1}} \leq \frac{1}{2^{k_{0}}},
$$

as $Y$ is injective on $A$ and $f$ is zero outside of $A$. Hence, $\int_{0}^{1} f(x) d x=0$ and any $y \in[0,1]$ with $f(y)=0$ yields $y \notin A$ by (3.5).

For the final item, the tangent and arctangent functions provide bijections between $(0,1)$ and $\mathbb{R}$. Hence, we may work with $A \subset \mathbb{R}$ and $Y: \mathbb{R} \rightarrow \mathbb{N}$ injective on $A$. We again consider $f$ as in (3.5), now as an $\mathbb{R} \rightarrow \mathbb{R}$-function. This function $f$ has bounded variation on any interval $[0, a]$ for $a>0$, in the same way as in the proof of Theorem 3.9. Since $e^{-z} \leq 1$ for $z \geq 0$, the previous paragraph yields $\int_{0}^{N} e^{-s t} f(t) d t=0$ for any $N \in \mathbb{N}, s \geq 0$, and hence $\mathfrak{L}(f)(s)=0$ for all $s \geq 0$. For 
$g: \mathbb{R} \rightarrow \mathbb{R}$ the zero everywhere function, we trivially have $\mathfrak{L}(g)(s)=0$ for any $s \geq 0$. Clearly, any $x \in[0,+\infty)$ such that $f(x)=g(x)=0$ also satisfies $x \notin A$, yielding a Cantor functional. The final sentence now follows in light of (the proof of) Corollary 3.10.

We note that theorem also goes through if we formulate the second item using the much weaker notions of quasi5 or cliquish 5 continuity in at least one point in $[0,1]$. These notions are found in e.g. [23, 28].

\section{REFERENCES}

[1] Jeremy Avigad and Solomon Feferman, Gödel's functional ("Dialectica") interpretation, Handbook of proof theory, Stud. Logic Found. Math., vol. 137, 1998, pp. 337-405.

[2] Michael J. Beeson, Foundations of constructive mathematics, Ergebnisse der Mathematik und ihrer Grenzgebiete, vol. 6, Springer, 1985.

[3] Errett Bishop, Foundations of constructive analysis, McGraw-Hill, 1967.

[4] E. Borel, Leçons sur la théorie des fonctions, Gauthier-Villars, Paris, 1898.

[5] Douglas Bridges and Fred Richman, Varieties of constructive mathematics, London Mathematical Society Lecture Note Series, vol. 97, Cambridge University Press, Cambridge, 1987.

[6] Douglas Bridges, A constructive look at functions of bounded variation, Bull. London Math. Soc. 32 (2000), no. 3, 316-324.

[7] Douglas Bridges and Ayan Mahalanobis, Bounded variation implies regulated: a constructive proof, J. Symbolic Logic 66 (2001), no. 4, 1695-1700.

[8] Douglas Bridges, Constructive Continuity of Increasing Functions, Beyond Traditional Probabilistic Data Processing Techniques: Interval, Fuzzy etc. Methods and Their Applications, 2020, pp. 9-19.

[9] L. E. J. Brouwer, Begründung der Mengenlehre unabhängig vom logischen Satz vom ausgeschlossenen Dritten. Erster Teil: Allgemeine Mengenlehre, Koninklijke Nederlandsche Akademie van Wetenschappen, Verhandelingen, 1ste sectie 12 (1918), no. 5, pp. 43.

[10] Wilfried Buchholz, Solomon Feferman, Wolfram Pohlers, and Wilfried Sieg, Iterated inductive definitions and subsystems of analysis, LNM 897, Springer, 1981.

[11] Fu Hua Chêng, On the rate of convergence of Bernstein polynomials of functions of bounded variation, J. Approx. Theory 39 (1983), no. 3, 259-274.

[12] Solomon Feferman, How a Little Bit goes a Long Way: Predicative Foundations of Analysis, 2013. unpublished notes from 1977-1981 with updated introduction, https://math.stanford.edu/ feferman/papers/pfa(1).pdf.

[13] Robert Gray, Georg Cantor and transcendental numbers, Amer. Math. Monthly 101 (1994), no. 9, 819-832.

[14] Noam Greenberg, Joseph S. Miller, and André Nies, Highness properties close to PA-completeness, To appear in Israel Journal of Mathematics (2019).

\footnotetext{
${ }^{5} \mathrm{~A}$ function $f: X \rightarrow \mathbb{R}$ is quasi-continuous (resp. cliquish) at $x \in X$ if for any $\epsilon>0$ and any open neighbourhood $U$ of $x$, there is a non-empty open ball $G \subset U$ with $(\forall y \in G)(|f(x)-f(y)|<\varepsilon)$ (resp. $(\forall y, z \in G)(|f(z)-f(y)|<\varepsilon))$.
} 
[15] Arend Heyting, Recent progress in intuitionistic analysis, Intuitionism and Proof Theory (Proc. Conf., Buffalo, N.Y., 1968), North-Holland, Amsterdam, 1970, pp. 95-100.

[16] David Hilbert and Paul Bernays, Grundlagen der Mathematik. II, Zweite Auflage. Die Grundlehren der mathematischen Wissenschaften, Band 50, Springer, 1970.

[17] Karel Hrbacek and Thomas Jech, Introduction to set theory, 3rd ed., Monographs and Textbooks in Pure and Applied Mathematics, vol. 220, Marcel Dekker, Inc., New York, 1999.

[18] Camillie Jordan, Sur la série de Fourier, Comptes rendus de l'Académie des Sciences, Paris, Gauthier-Villars 92 (1881), 228-230.

[19] Stephen C. Kleene, Recursive functionals and quantifiers of finite types. I, Trans. Amer. Math. Soc. 91 (1959), 1-52.

[20] Ulrich Kohlenbach, Foundational and mathematical uses of higher types, Reflections on the foundations of mathematics, Lect. Notes Log., vol. 15, ASL, 2002, pp. 92-116.

[21] _ On uniform weak König's lemma, Ann. Pure Appl. Logic 114 (2002), no. 1-3, 103-116. Commemorative Symposium Dedicated to Anne S. Troelstra (Noordwijkerhout, 1999).

[22] _ Higher order reverse mathematics, Reverse mathematics 2001, Lect. Notes Log., vol. 21, ASL, 2005, pp. 281-295.

[23] Marcin Kowalewski and Aleksander Maliszewski, Separating sets by cliquish functions, Topology Appl. 191 (2015), 10-15.

[24] Alexander P. Kreuzer, Bounded variation and the strength of Helly's selection theorem, Log. Methods Comput. Sci. 10 (2014), no. 4, 4:16, 15.

$[25] \_$Measure theory and higher order arithmetic, Proc. Amer. Math. Soc. 143 (2015), no. 12, 5411-5425.

[26] Kenneth Kunen, Set theory, Studies in Logic, vol. 34, College Publications, London, 2011.

[27] John Longley and Dag Normann, Higher-order Computability, Theory and Applications of Computability, Springer, 2015.

[28] T. Neubrunn, Quasi-continuity, Real Anal. Exchange 14 (1988/89), no. 2, 259306.

[29] André Nies, Marcus Triplett, and Keita Yokoyama, The reverse mathematics of theorems of Jordan and Lebesgue, J. Sym. Logic (2021), 1-18.

[30] Dag Normann and Sam Sanders, Nonstandard Analysis, Computability Theory, and their connections, Journal of Symbolic Logic 84 (2019), no. 4, 1422-1465.

[31] _ , The strength of compactness in Computability Theory and Nonstandard Analysis, Annals of Pure and Applied Logic 170 (2019), no. 11.

[32] cance of the uncountable, Journal of Mathematical Logic, https://doi.org/10.1142/S0219061319500016 (2019).

[33] - Representations in measure theory, Submitted, arXiv: https://arxiv.org/abs/1902.02756 (2019).

[34] - Open sets in Reverse Mathematics and Computability Theory, Journal of Logic and Computation 30 (2020), no. 8, pp. 40.

[35] _ Pincherle's theorem in reverse mathematics and computability theory, Ann. Pure Appl. Logic 171 (2020), no. 5, 102788, 41. 
[36] https://arxiv.org/abs/2007.07560 (2020), pp. 37.

[37] - The Axiom of Choice in Computability Theory and Reverse Mathematics, Journal of Logic and Computation 31 (2021), no. 1, 297-325.

[38] _ On robust theorems due to Bolzano, Weierstrass, and Cantor in Reverse Mathematics, See https://arxiv.org/abs/2102.04787 (2021), pp. 30.

[39] Fred Richman, Omniscience principles and functions of bounded variation, Mathematical Logic Quarterly 48 (2002), 111-116.

[40] Sam Sanders, Nets and Reverse Mathematics: initial results, LNCS 11558, Proceedings of CiE19, Springer (2019), 253-264.

[41] - Reverse Mathematics and computability theory of domain theory, LNCS 11541, Proceedings of WoLLIC19, Springer (2019), 550-568.

[42] _ Nets and Reverse Mathematics: a pilot study, Computability 10 (2021), no. 1, 31-62.

[43] Stephen G. Simpson, Subsystems of second order arithmetic, 2nd ed., Perspectives in Logic, CUP, 2009.

[44] J. Stillwell, Reverse mathematics, proofs from the inside out, Princeton Univ. Press, 2018.

[45] Alan Turing, On computable numbers, with an application to the Entscheidungs-problem, Proceedings of the London Mathematical Society 42 (1936), 230-265.

[46] Wim Veldman, Understanding and using Brouwer's continuity principle, Reuniting the antipodes (Venice, 1999), Synthese Lib., vol. 306, Kluwer, 2001, pp. 285-302.

[47] Klaus Weihrauch, Computable analysis, Springer-Verlag, Berlin, 2000.

[48] Xizhong Zheng and Robert Rettinger, Effective Jordan decomposition, Theory Comput. Syst. 38 (2005), no. 2, 189-209. 